\title{
Pulmonary Histoplasmosis
}

\author{
Carol A. Kauffman, MD
}

\author{
Address \\ Division of Infectious Diseases, Veterans Affairs Healthcare System, \\ 2215 Fuller Road, Ann Arbor, MI 48I05. \\ E-mail: ckauff@umich.edu \\ Current Infectious Disease Reports 200I, 3:279-285 \\ Current Science Inc. ISSN I523-3847 \\ Copyright $(2001$ by Current Science Inc.
}

Most patients with pulmonary histoplasmosis experience a self-limited illness that rarely requires treatment. However, patients who inhale a large burden of organisms from the environment and those who are immunosuppressed may develop severe, life-threatening pneumonia. Chronic histoplasmosis occurs almost exclusively in patients with severe chronic obstructive pulmonary disease. Complications of pulmonary histoplasmosis are mostly related to persistent mediastinal lymphadenopathy. Mild to moderate forms of pulmonary histoplasmosis should be treated with itraconazole. Patients with severe pulmonary involvement should initially receive amphotericin B; after the patient's condition has improved, therapy can be changed to itraconazole. Preventive measures should be used to protect workers from exposure to large numbers of Histoplasma capsulatum organisms.

\section{Introduction}

Histoplasma capsulatum is a dimorphic fungus that every year infects hundreds of thousands of individuals living in the Mississippi and Ohio River valleys and Central America. In its environmental phase, $H$. capsulatum exists as a mold. The organism grows profusely in dirt fertilized with the guano of birds and bats. Thus, areas that are frequented by migrating and roosting birds, old abandoned buildings, and caves often contain high concentrations of $H$. capsulatum. Infection is common in endemic areas, and most persons have been infected before adulthood.

The conidia (spores) are aerosolized and inhaled into the lungs, where they cause primary pulmonary infection. The extent of disease is determined by the number of conidia that are inhaled and the host's immune response. Thus, a small inoculum may cause severe pulmonary infection or progress to disseminated histoplasmosis in a host whose cell-mediated immune system cannot contain the organism ( $e g$, a patient with AIDS). On the other hand, a healthy person may develop severe, life-threatening pulmonary infection if a large number of conidia are inhaled. Such an event might occur during demolition activities, spelunking in a heavily infested cave, or renovations on structures, such as bridges and old buildings.

Not surprisingly, the predominant site of symptomatic infection is the lungs. However, infection is asymptomatic in most persons who have been infected. Most patients with symptomatic infection have a self-limited illness that is usually not diagnosed as histoplasmosis and is not treated with antifungal agents. Although most individuals have this benign form of pulmonary histoplasmosis, acute severe pneumonia and chronic cavitary infection occur in appropriate hosts and can be fatal if not treated. Complications of pulmonary histoplasmosis include pericarditis, mediastinal granuloma, and mediastinal fibrosis (Table 1).

Even though the initial and often the only manifestations of histoplasmosis are pulmonary, it is important to remember that histoplasmosis is a systemic reticuloendothelial disease for which the primary host defense mechanism is cell-mediated immunity. Most persons who have been infected with $H$. capsulatum have had asymptomatic dissemination; however, only rarely does this event culminate in symptomatic acute or chronic disseminated histoplasmosis.

\section{Acute Pulmonary Histoplasmosis Acute self-limited histoplasmosis}

Symptomatic acute pulmonary histoplasmosis is characterized by fever, chills, cough (usually nonproductive), myalgias, arthralgias, and chest pain that is made worse by coughing. Chest radiography shows patchy lobar or multilobe nodular infiltrates [1].

Acute pulmonary histoplasmosis is most easily diagnosed when the patient has been involved in an outbreak [2•]. For individual cases, which occur sporadically, the diagnosis is usually not entertained until the patient does not respond to several courses of antibiotics. A careful history of the patient's activities for several weeks before symptom onset may point to the possibility of fungal pneumonia. Outdoor and spelunking activities in the endemic area should prompt suspicion of histoplasmosis. Most difficult to differentiate from acute pulmonary histoplasmosis is acute pulmonary blastomycosis, which is acquired in many of the same regions of the United States as histoplasmosis. Also included in the differential diagnosis are atypical pneumonias due to Mycoplasma, Legionella, and Chlamydia species. Hilar or mediastinal lymphadenopathy are common with histoplasmosis but uncommon with these atypical pneumonias. 


\section{Table I. Classification scheme for pulmonary histoplasmosis}

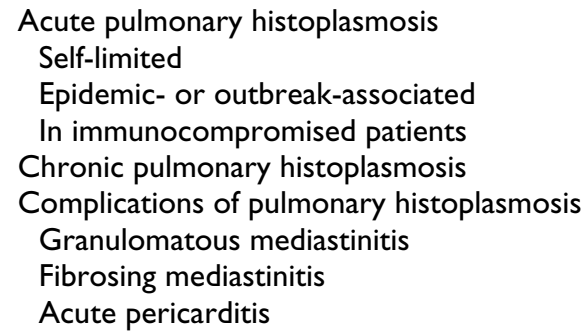

Generally, symptoms resolve over several weeks, although lymphadenopathy may persist for weeks to months. Symptoms can recur, and a small number of patients will have persistent or relapsing fatigue for months after pneumonia has resolved.

\section{Epidemic or outbreak-associated histoplasmosis}

This form of acute pulmonary histoplasmosis occurs when a large number of conidia are inhaled. Epidemiologic investigation often reveals a discrete point source that is heavily contaminated with $H$. capsulatum. Most often, several patients with symptoms of varying severity, depending on exposure to the point source, seek medical attention $[2 \bullet, 3]$. Although most patients have primary infection, reinfection histoplasmosis has been documented when the conidial burden is high. Symptoms include high spiking fever, prostration, dyspnea, and cough. Diffuse nodular infiltrates are usually noted (Fig. 1); acute respiratory distress syndrome can develop in severe cases. After resolution of infection, the chest radiograph may show multiple calcified nodules (Fig. 2).

\section{Acute pulmonary histoplasmosis in immunocompromised hosts}

In patients with cell-mediated immune deficiencies, including those who have advanced HIV infection, have a hematologic malignancy, have received a transplant, or are receiving immunosuppressive medications, pulmonary infection is more severe than in normal hosts [4,5]. Although pulmonary infection may be the only manifestation of histoplasmosis, in most immunosuppressed patients pulmonary involvement is merely one component of widespread dissemination. Prostration, fever, chills, and sweats are prominent; marked dyspnea and hypoxemia can progress quickly to acute respiratory distress syndrome. Chest radiography shows diffuse bilateral infiltrates (Fig. 3). The most important diagnostic procedure is bronchoscopy, which is done to provide tissue for histopathology and culture unless histoplasmosis is confirmed by culture of blood, detection of $H$. capsulatum antigen in urine or serum, or biopsy and culture of other involved tissues.

\section{Chronic Pulmonary Histoplasmosis}

The typical patient with chronic histoplasmosis is an older man with chronic obstructive pulmonary disease. The symptoms usually are fever, fatigue, anorexia, weight loss, cough that produces purulent sputum, and hemoptysis $[6,7]$. On chest radiography, infiltrates are unilateral or bilateral, are almost always in the upper lobes, and are cavitary (Fig. 4). Extensive scarring, thought to be related to the host's response to $H$. capsulatum antigens, occurs in the lower lobes. Pleural involvement is uncommon.

Chronic pulmonary histoplasmosis mimics tuberculosis in terms of symptoms, signs, and radiographic findings. Also presenting a similar clinical picture are other chronic fungal pneumonias (including blastomycosis, sporotrichosis, and coccidioidomycosis) and nontuberculous mycobacterial infections. Microbiologic studies confirm the correct diagnosis.

\section{Complications of Pulmonary Histoplasmosis Granulomatous mediastinitis}

This is an uncommon complication of pulmonary histoplasmosis. Most patients with acute pneumonia have associated mediastinal lymphadenopathy, which resolves within a few months after resolution of pneumonia. Patients with mediastinal granuloma have persistent lymphadenopathy, often involving multiple nodal groups $[8,9]$. The enlarged nodes can erode into the lumen of the esophagus, lead to traction diverticula, or cause tracheoesophageal fistula formation. Compression of a bronchus can lead to intermittent obstruction and pneumonia; rarely, the nodes spontaneously drain into adjacent soft tissues of the neck.

Chest radiography reveals enlarged hilar and mediastinal lymph nodes. The extent of lymphadenopathy is best demonstrated by CT. Areas of necrosis and calcium deposition are commonly noted on this imaging test. Most of these patients have a self-limited process and do not develop fibrosing mediastinitis. Mediastinoscopy and biopsy often reveal caseous material, which may contain a few yeastlike organisms typical for H. capsulatum.

\section{Fibrosing mediastinitis}

This rare complication of pulmonary histoplasmosis is particularly difficult to manage. For unknown reasons, the host, usually a young adult, responds to infection in the mediastinal lymph nodes with an excessive amount of fibrosis $[10,11]$. The organism is rarely seen histopathologically and rarely grows in culture of the tissue. The exuberant fibrous tissue entraps the great vessels, causing respiratory distress, heart failure, pulmonary emboli, and superior vena cava syndrome. When the fibrous tissue entraps the esophagus, odynophagia is prominent; constriction of the 

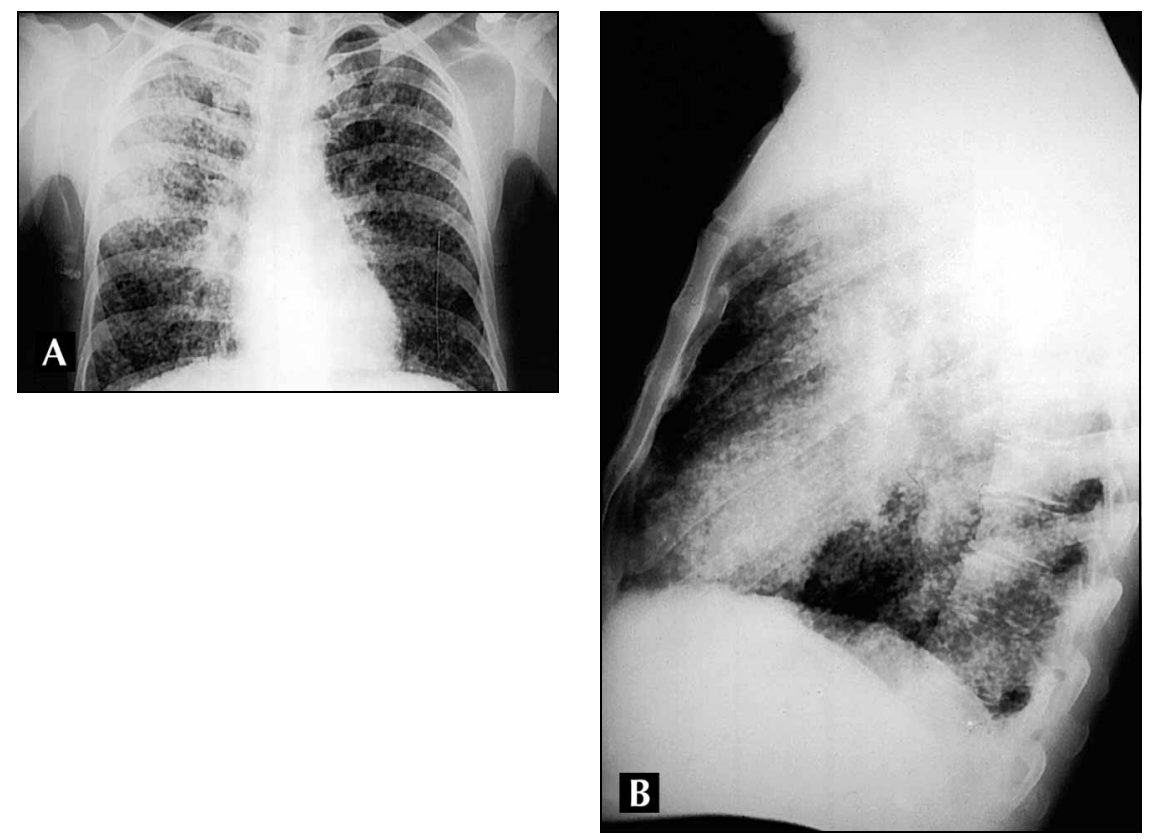

Figure 1. Posterior-anterior (A) and lateral (B) chest radiographs of a painter who scraped off a large amount of material that had accumulated for years on a bridge abutment that served as a pigeon roost. Extensive bilateral pulmonary infiltrates composed of various-sized nodules with confluence in the right upper lung fields are seen. The patient was acutely ill with fever and hypoxemia.

bronchi results in dyspnea, cough, wheezing, and hemoptysis. No effective therapy is available for this progressive disease, and no evidence suggests that antifungal agents or corticosteroids improve the outcome.

\section{Pericarditis}

Pericarditis is another uncommon complication of acute pulmonary histoplasmosis $[12,13]$. Pericardial involvement presumably reflects an inflammatory response to pulmonary infection; $H$. capsulatum has rarely been isolated from pericardial fluid, and the syndrome is almost always self-limited. Pleural effusions and mediastinal lymphadenopathy are commonly associated with pericarditis. The pericardial fluid is exudative and usually bloody, resembling fluid seen with tuberculous pericarditis. Although symptoms generally resolve after several months, relapses are not uncommon. Drainage is required if tamponade is imminent, but this is a rare complication. The long-term outcome is excellent; only rarely has constrictive pericarditis been reported.

\section{Diagnosis \\ Culture}

The definitive diagnostic test for histoplasmosis is growth of the organism in culture; unfortunately, culture may take up to 6 weeks. For an acutely ill person, this is obviously not helpful other than for later confirmation of a suspected diagnosis of histoplasmosis. Sputum, bronchoalveolar lavage fluid, and lung or mediastinal lymph node biopsy specimens should be sent to the microbiology laboratory for culture. Blood or other tissues, such as bone marrow, liver, or mucocutaneous lesions, prove useful for culture only among patients with evidence of dissemination. The laboratory should be informed when pulmonary histoplasmosis is a diagnostic consideration so that special media can be used for the culture of pulmonary samples. Such precautions are necessary to discourage the growth of commensal fungi, such as Candida species, whose rapid growth prevents the emergence of the slowly growing $H$. capsulatum. Newly developed DNA probes specific for $H$. capsulatum have markedly improved the identification of the organism within a short time after growth has begun [14].

\section{Antibody assays}

Serology plays an important adjunctive role in the diagnosis of histoplasmosis $[14,15]$. Both complement fixation and immunodiffusion tests are available; both are modestly sensitive ( $75 \%$ to $85 \%)$, but immunodiffusion is more specific than complement fixation (> $95 \%$ vs $85 \%$ to $90 \%$ ). Most problems with false-negative serologic test results occur in immunosuppressed patients who cannot mount an antibody response. Most patients with pulmonary histoplasmosis are not overtly immunosuppressed, and thus serologic tests can be helpful. In a patient with possible acute pulmonary histoplasmosis, a documented fourfold increase in complement fixation titer or the appearance of an $M$ precipitin band on the immunodiffusion assay establishes the diagnosis of histoplasmosis. Patients with chronic cavitary pulmonary histoplasmosis almost always have an elevated complement fixation titer $(>1: 32)$ and may have only an $\mathrm{M}$ band or both $\mathrm{H}$ and $\mathrm{M}$ precipitin bands by immunodiffusion assay. A positive result on serologic testing with a typical clinical picture for chronic cavitary pulmonary histoplasmosis allows initiation of treatment while results of sputum cultures are pending. Serologic tests are less definitive in patients with mediastinal lymphadenopathy or fibrosis, and results should be confirmed by biopsy of involved tissue. 


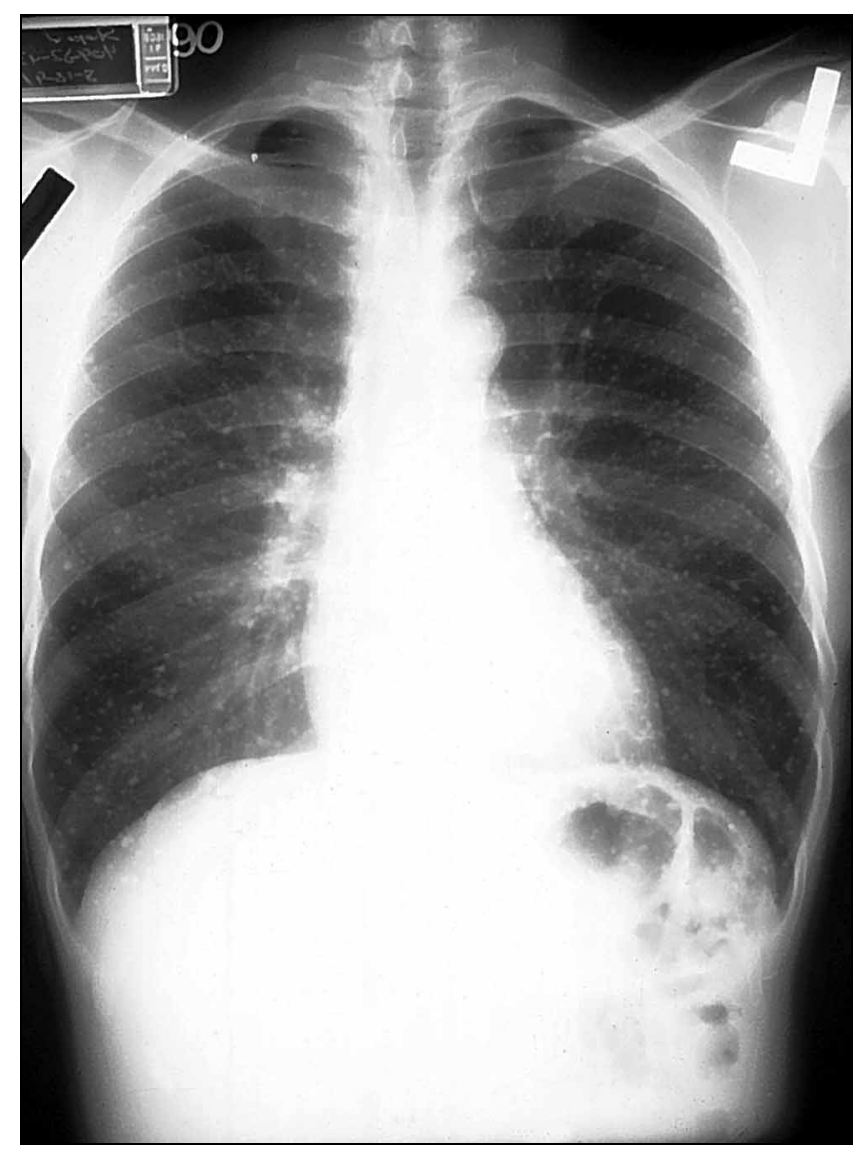

Figure 2. Resolution of acute epidemic histoplasmosis with multiple calcified nodules seen diffusely throughout both lung fields.

\section{Antigen assays}

Tests for $H$. capsulatum polysaccharide antigen, which are extremely helpful in patients with AIDS who have disseminated infection and a large burden of $H$. capsulatum, are less useful for pulmonary forms of histoplasmosis [16,17]. Antigen is detected in only about $20 \%$ of patients with pulmonary histoplasmosis. This test is not sensitive for patients with a low burden of organisms; although the test was initially thought to be highly specific, cross-reactivity has been reported rarely in patients with blastomycosis, paracoccidioidomycosis, and penicilliosis [18]. An enzyme immunoassay has recently replaced the original radioimmunoassay, improving the ease and safety of the assay [19]. Rapid DNA-based techniques that would allow a fast and specific diagnosis of histoplasmosis are not available.

\section{Histopathology}

Biopsy evidence of the yeast phase of $H$. capsulatum is helpful in the diagnosis of histoplasmosis. The organisms appear as distinctive, fairly uniform, 2- to 4- $\mu \mathrm{m}$ oval budding yeasts. However, tissue biopsy is most useful in patients with disseminated disease; bone marrow, liver, and mucocutaneous lesions often reveal the organisms. Biopsy is less useful in pulmonary histoplasmosis. For most patients with acute pulmonary histoplasmosis, biopsy is not indicated unless they are severely ill. Among patients with chronic pulmonary histoplasmosis or granulomatous mediastinitis, biopsy of lung or lymph nodes may reveal the organism; for those with fibrosing mediastinitis, it is highly unlikely that organisms will be seen. Biopsy material must be stained with methenamine silver or periodic acid-Schiff stains in order to visualize the organisms; routine hematoxylin and eosin staining does not show the tiny $H$. capsulatum yeasts. Even with special staining procedures, finding the tiny yeast forms, which often are present in low numbers, demands careful timeconsuming perusal of the material. In contrast to the situation with larger yeasts, such as Blastomyces dermatitidis, it is extremely unusual to find $H$. capsulatum on cytologic examination of sputum or bronchoalveolar lavage fluid.

\section{Skin tests}

Histoplasmin skin testing is not useful for diagnosing histoplasmosis in individual patients, although it has been used in the past to define the epidemiology of histoplasmosis. In the endemic area, as many as $85 \%$ of adults are skin test positive because of previous exposure to the organism, test results are negative in patients who have severe infection, and cross-reactions occur in patients with other fungal infections, especially blastomycosis.

\section{Treatment}

The Infectious Diseases Society of American recently defined guidelines for treatment of pulmonary histoplasmosis [20••]. In most patients with pulmonary histoplasmosis, itraconazole is the drug of choice. Amphotericin B is required for patients who have severe acute pulmonary histoplasmosis and those who cannot tolerate or do not absorb itraconazole or are taking other medications that preclude the use of itraconazole. Fluconazole should be considered a second-line agent because compared with itraconazole, primary response rates are lower for patients with and those without AIDS and relapse rates are higher in patients with AIDS [21-25].

Treatment is generally not recommended for patients with acute pulmonary histoplasmosis; indeed, the condition is often not diagnosed until after the patient's symptoms have resolved. However, if the diagnosis has been established and the patient is still symptomatic after 4 weeks, therapy with itraconazole, $200 \mathrm{mg}$ once or twice daily for 6 to 12 weeks, is recommended.

In many patients with epidemic histoplasmosis, pneumonia will resolve without treatment; however, those with severe pulmonary involvement should be treated with amphotericin B. Similarly, immunosuppressed hosts with severe pneumonia should initially receive amphotericin $\mathrm{B}$. Although the intravenous formulation of itraconazole is likely to be effective [26], no data are available for patients with life-threatening infection; as a result, the drug cannot be recommended in this setting. After an initial favorable 


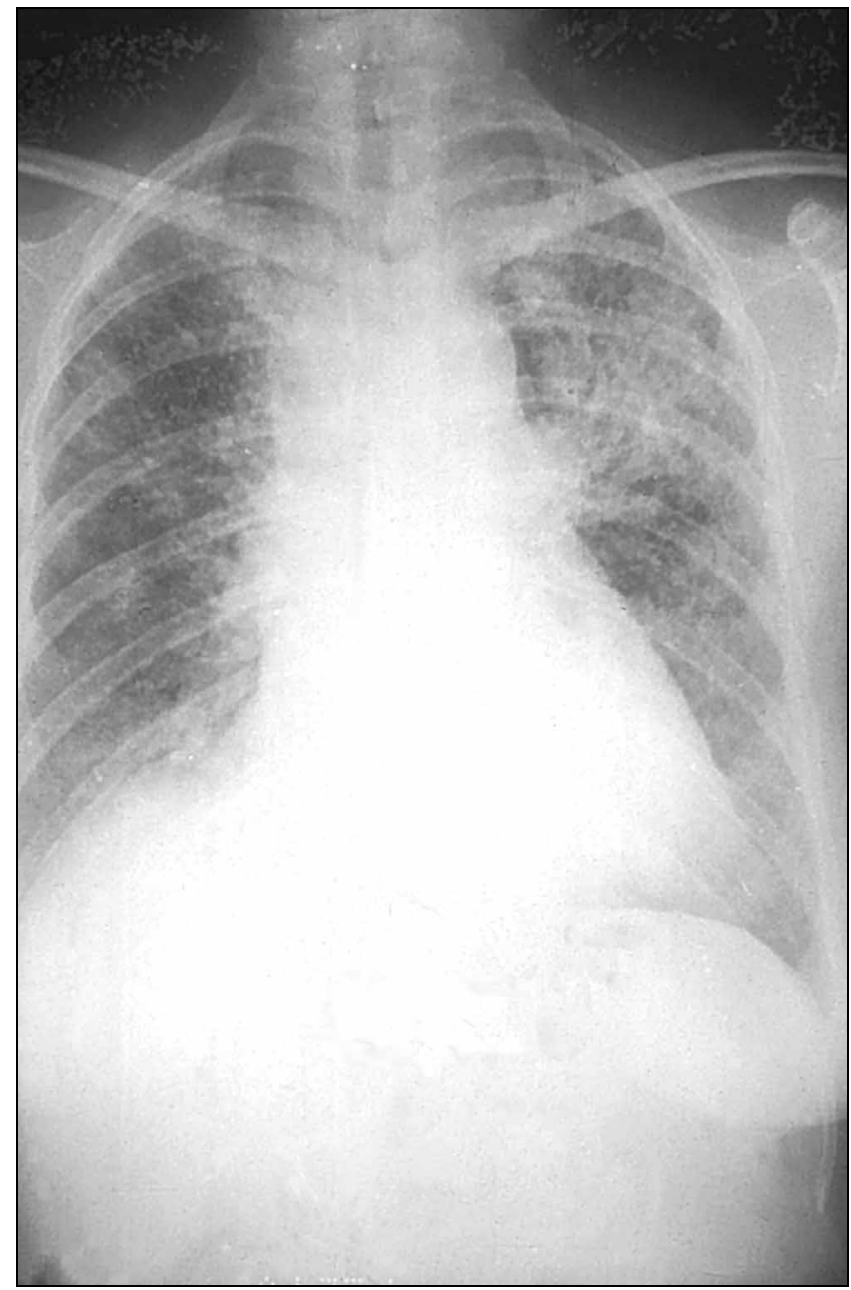

Figure 3. Diffuse pulmonary infiltrates due to acute pulmonary histoplasmosis in a young woman who had received a renal transplant several months previously. Bronchoscopy yielded the diagnosis, and she responded rapidly to therapy with amphotericin B.

response to amphotericin $\mathrm{B}$ and after the patient's condition is stable, treatment can be changed to oral itraconazole $[20 \bullet \bullet$. Corticosteroids have been advocated as a means to dampen the immune response of patients with epidemic histoplasmosis [27]. However, corticosteroids should never be used without a concomitant antifungal agent.

Therapy is required for patients with chronic pulmonary histoplasmosis because the fatality rate is close to $100 \%$ without antifungal treatment [6]. Itraconazole is almost always the drug used in this circumstance because the course of the infection is subacute to chronic. Treatment should be continued for 12 to 24 months. Even with appropriate antifungal therapy, outcomes are poor, mostly because of the severe emphysema present in these patients.

No studies have proved that antifungal therapy is beneficial for granulomatous mediastinitis. However, patients may benefit from antifungal therapy because active infection is probably causing the continued lymphadenopathy.
Most clinicians prescribe itraconazole unless the patient has severe obstructive complications, which would mandate use of amphotericin B. Very little evidence suggests that treating patients who have fibrosing mediastinitis with antifungal agents has any benefit [10]; however, since the outcome of this disease is so dismal, most patients will have received therapy with itraconazole for several months because of the slim possibility that it may provide some benefit. Pericarditis should be treated with nonsteroidal anti-inflammatory agents (or corticosteroids for severe cases), not with antifungal agents.

\section{Prevention}

Histoplasmosis is exceedingly common in endemic areas. Preventive measures should focus on avoiding high-risk behaviors likely to be associated with exposure to the organism, or taking protective measures to prevent infection if these activities are necessary. A recent publication from the National Institute for Occupational Safety and Health (NIOSH) and the National Center for Infectious Diseases (NCID) (part of the Centers for Disease Control and Prevention) gives explicit directions regarding the safety of workers involved in risky behaviors, such as removing debris from or demolishing old buildings or structures likely to be heavily contaminated with $H$. capsulatum [28]. Workers must be provided with appropriate respirator protection; surgical masks are not effective. Dust suppression techniques using water sprays during demolition should be employed, and specialized equipment may be necessary for the safe removal of large accumulations of potentially contaminated bird and bat excreta. Before an activity likely to result in exposure to H. capsulatum is undertaken, advice should be sought from NIOSH or NCID. Immunocompromised persons, including those with HIV infection, those with hematologic malignancies, and transplant recipients, should not indulge in spelunking or undertake renovation projects that involve soil or buildings that could be contaminated with H. capsulatum.

A recent blinded randomized trial conducted by the Mycoses Study Group showed that prophylactic itraconazole can prevent histoplasmosis in patients with AIDS whose CD4 counts are less than 150 cells $/ \mathrm{mm}^{3}$ [29••]. In endemic areas experiencing moderately high rates of infection $(>5$ cases/100 patient-years), $200 \mathrm{mg}$ of itraconazole daily should be given to patients with AIDS whose CD4 counts are less than 150 cells $/ \mathrm{mm}^{3}$. However, recent trends show that almost all patients with AIDS who have histoplasmosis have not received previous medical care and present with histoplasmosis as their AIDS-defining illness. Thus, the current population at most risk cannot be targeted for prophylaxis. At the other end of the spectrum are patients who have faithfully taken and responded to highly active antiretroviral therapy; prophylaxis has little role in this population because the risk of histoplasmosis is now quite low. 


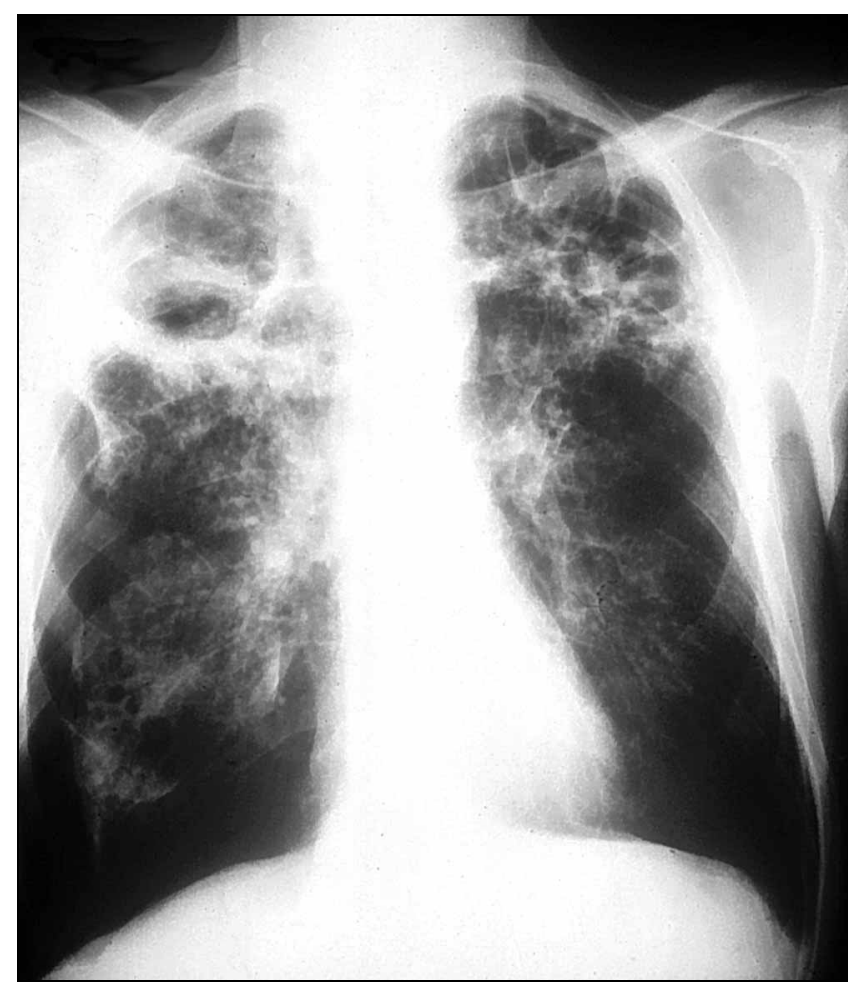

Figure 4. Chronic cavitary pulmonary histoplasmosis in an older man with severe chronic obstructive pulmonary disease. Multiple upper lobe cavities are present in both lungs. Despite therapy with ketoconazole and clearing of Histoplasma capsulatum from his sputum, he experienced progressive pulmonary insufficiency.

\section{Conclusions}

Histoplasmosis is an infection of the reticuloendothelial system, but the primary disease manifestations are usually associated with infection at the portal of entry, the lungs. Although most symptomatic patients have only self-limited pneumonia, immunosuppressed hosts and healthy hosts who inhale many organisms can develop severe pneumonia, and patients with emphysema develop chronic progressive pulmonary infection. Complications of pulmonary histoplasmosis most often are secondary to infection of mediastinal lymph nodes and include granulomatous mediastinitis, fibrosing mediastinitis, and acute pericarditis.

The newest aspects of diagnosis include molecular assays for rapid confirmation of $H$. capsulatum growth in vitro and use of enzyme immunoassay to detect $H$. capsulatum antigen; however, enzyme immunoassay is more useful for disseminated histoplasmosis than for pulmonary histoplasmosis. In the past decade, multicenter trials have defined appropriate treatment regimens for the various forms of pulmonary histoplasmosis. Recently, the Infectious Diseases Society of America published guidelines for the treatment of histoplasmosis based on the results of these trials and reviews of clinical experience.

Excluding self-limited acute pulmonary histoplasmosis, which generally does not require treatment, itraconazole is the appropriate antifungal agent for most patients with mild to moderate acute or chronic pulmonary histoplasmosis. Amphotericin B should be used for initial therapy among patients who have severe life-threatening pulmonary infection; after the patient's condition has improved, therapy can then be switched to itraconazole.

Finally, the Centers for Disease Control and Prevention has recently issued preventive measures to avoid exposure to $H$. capsulatum by workers performing high-risk activities.

\section{References and Recommended Reading}

Papers of particular interest, published recently, have been highlighted as:

- Of importance

- Of major importance

1. Gurney JW, Conces DJ Jr: Pulmonary histoplasmosis. Radiology 1996, 199:297-306.

2. Wheat J: Histoplasmosis. Experience during outbreaks in Indianapolis and review of the literature. Medicine(Baltimore) 1997, 76:339-354.

This overview of histoplasmosis gives the perspective of a recognized authority who probably has the most extensive experience in diagnosis and treatment of histoplasmosis.

3. Stobierski MG, Hospedales CJ, Hall WN, et al:: Outbreak of histoplasmosis among employees in a paper factoryMichigan 1993. J Clin Microbiol 1996, 34:1220-1223.

4. Wheat LJ, Connolly-Stringfield PA, Baker RL, et al.: Disseminated histoplasmosis in the acquired immune deficiency syndrome: clinical findings, diagnosis and treatment, and review of the literature. Medicine (Baltimore) 1990, 69:361-374.

5. Kauffman CA, Israel KS, Smith JW, et al.: Histoplasmosis in immunosuppressed patients. Am J Med 1978, 64:923-932.

6. Goodwin RA Jr, Owens FT, Snell JD, et al.: Chronic pulmonary histoplasmosis. Medicine (Baltimore) 1976, 55:413-452.

7. Wheat LJ, Wass J, Norton J, et al.: Cavitary histoplasmosis occurring during two large urban outbreaks: analysis of clinical, epidemiologic, roentgenographic, and laboratory features. Medicine (Baltimore) 1984, 63:201-209.

8. Landay MJ, Rollins NK: Mediastinal histoplasmosis granuloma: evaluation with CT. Radiology 1989, 172:657-659.

9. Savides TJ, Gress FG, Wheat LJ, et al.: Dysphagia due to mediastinal granulomas: diagnosis with endoscopic ultrasonography. Gastroenterology 1995, 109:366-373.

10. Loyd JE, Tillman BF, Atkinson JB, et al.: Mediastinal fibrosis complicating histoplasmosis. Medicine (Baltimore) 1988, 67:295-310.

11. Mathisen DJ, Grillo HC: Clinical manifestations of mediastinal fibrosis and histoplasmosis. Ann Thorac Surg 1992, 54:1053-1058.

12. Picardi JL, Kauffman CA, Schwarz J, et al.: Pericarditis caused by Histoplasma capsulatum. Am J Cardiol 1976, 37:82-88.

13. Wheat $\mathrm{LJ}$, Stein $\mathrm{L}$, Corya $\mathrm{BC}$, et al.: Pericarditis as a manifestation of histoplasmosis during two large urban outbreaks. Medicine (Baltimore) 1983, 62:110-119.

14. Wheat LJ: Diagnosis and management of histoplasmosis. Eur J Clin Microbiol Infect Dis 1989, 8:480-490.

15. Wheat LJ: Histoplasmosis in Indianapolis. Clin Infect Dis 1992, 14(suppl 1):S91-S99.

16. Wheat LJ, Kohler RB, Tewari RP: Diagnosis of disseminated histoplasmosis by detection of Histoplasma capsulatum antigen in serum and urine specimens. N Engl J Med 1986, 314:83-88.

17. Wheat LJ, Connolly-Stringfield P, Kohler RB, et al.: Histoplasma capsulatum polysaccharide antigen detection in the diagnosis and management of disseminated histoplasmosis in patients with acquired immunodeficiency syndrome. Am J Med 1989, 89:396-400. 
18. Wheat $\mathrm{J}$, Wheat $\mathrm{H}$, Connolly $\mathrm{P}$, et al.: Cross-reactivity in Histoplasma capsulatum variety capsulatum antigen assays of urine samples from patients with endemic mycoses. Clin Infect Dis 1997, 24:1169-1171.

19. Durkin MM, Connolly PA, Wheat LJ: Comparison of radioimmunoassay and enzyme-linked immunoassay methods for detection of Histoplasma capsulatum var. capsulatum antigen. J Clin Microbiol 1997, 35:2252-2255.

$20 . \bullet$ Wheat J, Sarosi G, McKinsey D, et al.: Practice guidelines for the management of patients with histoplasmosis. Clin Infect Dis 2000, 30:688-695.

An important report on treatment options for all forms of histoplasmosis.

21. Dismukes WE, Bradsher RW Jr, Cloud GC, et al.: Itraconazole therapy for blastomycosis and histoplasmosis. NIAID Mycoses Study Group. Am J Med 1992, 103:223-232.

22. Wheat J, Hafner R, Korzun AH, et al.: Itraconazole treatment of disseminated histoplasmosis in patients with the acquired immunodeficiency syndrome. AIDS Clinical Trial Group. Am J Med 1995, 98: 336-342.

23. Wheat J, Hafner R, Wulfson M, et al.: Prevention of relapse of histoplasmosis with itraconazole in patients with the acquired immunodeficiency syndrome. Ann Intern Med 1993, 118:610-616
24. McKinsey DS, Kauffman CA, Pappas PG, et al.: Fluconazole therapy for histoplasmosis. Clin Infect Dis 1996, 23:996-1001.

25. Wheat J, MaWhinney S, Hafner R, et al:: Treatment of histoplasmosis with fluconazole in patients with acquired immunodeficiency syndrome. Am J Med 1997, 103:223-232.

26. Stevens DA: Itraconazole in cyclodextrin solution. Pharmacotherapy 1999, 19:603-611.

27. Kataria YP, Campbell PB, Burlingham BT: Acute pulmonary histoplasmosis presenting as adult respiratory distress syndrome: effect of therapy on clinical and laboratory features. South Med J 1981, 74:534-537.

28. McKinsey DS, Wheat LJ, Cloud GA, et al.: Itraconazole prophylaxis for fungal infections in patients with advanced human immunodeficiency virus infection: randomized, placebo-controlled, double-blind study. Clin Infect Dis 1999, 28:1049-1056.

29.• Lenhart SW, Schafer MP, Singal M, et al.: Histoplasmosis: Protecting Workers at Risk. Bethesda, MD: Department of Health and Human Services; 1997. DHHS (NIOSH) Publication No. 97-146.

Well-written, practical guide on how to protect workers and others at risk for extensive exposure to $H$. capsulatum. 